\title{
Cognition as Orientation in the Environment ${ }^{6}$
}

\author{
Andrzej Lewicki (1960) \\ translated by Magdalena Kopczyńska
}

There is one major reason that the conception of nervous reflection cannot be directly associated with "cognition" even though it undoubtedly should be a vital component of the definition of this term. Namely, reflection, understood as the creation of equivalents of external stimuli, is a process that happens not only in the brains of living creatures, but also in inanimate matter. A thermometer "reflects" changes in temperature, but we would not say that it "knows" themit is the man who knows the temperature when using a thermometer. Reflection means cognition only when it determines offensive or defensive reactions of an organism: when it constitutes an element of the mechanism of adaptation to the environment, it enables the individual to be guided by the reflected external phenomena. [...]

Keywords: cognition; indication of value; indication pointing to action; Lewicki Andrzej; Pavlov Ivan; orientation in the environment; tendency

\section{The conception of orientation in the environment}

There is one major reason that the [...] conception of nervous reflection cannot be directly associated with "cognition" even though it undoubtedly should be a vital component of the definition of this term. Namely, reflection, understood as the creation of equivalents of external stimuli, is a process that happens not only in the brains of living creatures, but also in inanimate matter. A thermometer "reflects" changes in temperature, but we would not say that it "knows" them-it is the man who knows the temperature when using a thermometer. Reflection means cognition only when it determines offensive or defensive reactions of an organism: when it consti-

\footnotetext{
${ }^{6}$ Translation of Chapter VI of the book Procesy poznawcze $i$ orientacja $w$ otoczeniu [The Cognitive Processes and Orientation in the environment] by Andrzej Lewicki (1960), Warsaw: Państwowe Wydawnictwo Naukowe. The translation is published with the kind permission of the copyright Owner.
} 
tutes an element of the mechanism of adaptation to the environment, it enables the individual to be guided by the reflected external phenomena. The thermometer will "reflect" even the highest temperatures passively until it is destroyed, but, when reflecting higher temperatures, an animal will react defensively to them, trying to withdraw from the unfavorable circumstances, which is why in the case of an animal we are inclined to say it "knows" the temperature. In other words, knowing is not merely reflection; it must comprise a perception of the values of the phenomena reflected and a perception of the action that should be performed by an individual in a given situation in order to maintain inner balance. Seemingly, reflection is just a means by which a living creature "understands" the positive or negative significance of phenomena for their life and their own future action in relation to these phenomena; only this entire process of "cognition" explains how one is guided by relevant factors, which further means that it, and not only the very reflection, deserves to be termed "cognition". We discussed these questions in Chapter IV when analyzing the cognitive process as a mechanism of following the features of external objects (see p. 101).

Instead of the term "understanding", which is rather imprecise and traditionally limited to the facts of consciousness, I will describe the cognitive process using a different term which is also quite popular but less questionable: orientation in the environment. This term is often used in colloquial speech when we want to specify how efficiently a person solves theoretical or practical tasks entrusted to them. We say that a student has better or worse orientation, for example in mathematics, meaning that this student solves mathematical tasks more or less correctly, but we also assign a good sense of direction (translator's note: "orientation” in Polish) to a tourist who can find their way and a bad sense of direction to one who becomes lost. This term is not burdened with the traditions of the psychology of consciousness to the same degree as "understanding" and can be freely used in reference to both humans and animals. For instance, Dembowski mentions the orientation of rats in a maze (1946a, pp. 344, 352) and elaborates on the properties of a field that enable bees to find their way back to the hive (loc. cit., p. 255f.), etc. Even Pavlov does not hesitate to use this conception in relation to dogs, considering it a totally objective term that does not refer to consciousness. Initially, he was of the opinion that only the reflexive, unconditioned mechanism of adaptation deserves to be called "orientation in the environment” (1952, p. 77), but he later extended the meaning of the term considerably and distinguished three levels of orientation: instinctive orientation, i.e. reflexive-unconditioned orientation; primary reflexive-conditioned orientation; and secondary verbal orientation (1952, p. 497). 
The earlier analysis of the facts of animals being guided by features of objects (see Chapter IV, p. 101f.) makes it possible to specify how the term "orientation in the environment" should be understood. Namely, it must be acknowledged that the basic component of the orientation process is the reflection of the features of an object or-to be more general-the phenomena that are the determinants of the value of the object. I will refer to this component as orientation in value. Orientation in the value of objects found in the environment should be recognized as the principal component of the orientation process because it must happen in each act of adaptation: adaptation to the environment consists in the fact that an animal reacts proportionally to the value a given object has for it, which means that it must somehow perceive this value and somehow orient itself in it.

However, the very orientation in the value of an object does not normally suffice for the animal to have its conduct guided by a given determinant of value - to appropriate a valuable object or defend itself against damage. For this purpose, it is necessary to perform an appropriate a ction that is guided by a given indication of value. Only in certain laboratory experiments, such as Pavlov's experiments in digestive conditioned reflexes, is it enough when the dog calmly stands in the harness and salivates so that the food literally gets into its mouth. Such "passive" orientation in the environment is clearly an artificial phenomenon; in normal circumstances orientation in value has to be related to an activity that is adapted to a particular value-a positive, offensive one if the value is positive and a negative one if the object poses a threat to the animal. In the simplest cases, the indication of the value itself will determine the action the animal should undertake. Usually this happens when the reaction is innate or instinctive; for example, when the animal attempts to escape or attacks to avoid being injured. However, in other cases the action taken requires that the animal should be guided by different indications. A typical example is experiments in which an animal sees a known bait (the sight of bait, such as a fruit, is an indication of value) but is separated from it by an obstacle, the overcoming of which demands a separate action and orientation in various features of the situation: opening the door of the cage, pulling a bait with a stick, opening a box containing a bait, etc. In all these cases, a separate component must be added to orientation in the value of an object. I will describe this as orientation in an action adapted to a given situation on the basis of the reflection of separate indications pointing to action.

Thus, it follows that there are two major types of orientation processes: a simpler one, comprising only the reflection of the indication of value and usually an inherent reaction (secretory, motoric or combined), and a more complex one, including reflection of separate indications pointing to action. 
However, if we define "cognition" as orientation in the environment, i.e. a reflection of indications of value and indications pointing to action adapted to the situation, we should ask in which terms we are supposed to describe the phenomena of perceiving value and "working out" the action that combine with reflection in order to transform it into a cognitive process. We should require that these terms and "reflection" itself should also be defined so that "orientation in the environment" would be a conception superior to the terms used in the psychology of consciousness and could be applied where we do not have an opportunity to have insight into one's consciousness. This means these processes should be considered as activities of the central nervous system without smuggling into our definition terms from the psychology of consciousness such as "value assessment" or "working out the action"; otherwise, "orientation in the environment" would cease to be an appropriate tool for the analysis of the mechanism of behavior, but it would force us to feel certain states of consciousness in examined individuals.

\section{Orientation in value as a component of the reflexive mechanism}

By saying that objects or situations in the environment have one "value" or another for an individual, we may mean two different things. Firstly, we can indicate "biological value", i.e. the objective benefit that external stimuli have for the health and life of an animal. We assess this value based on our knowledge of what is healthy or harmful for a given organism, irrespective of how one behaves towards certain objects. We know which type of food is valuable for a particular animal species because observation has shown that this food does this species good. Similarly, we can assess the threats various situations pose for different species: we know that extended immersion in water is fatal for animals that breathe with lungs; we know which enemies representatives of particular species might encounter, etc. Secondly, "value" can be understood as "behavioral value". In this context, what has value for an animal are those objects and situations that elicit reactions in it: if the reaction is positive, we can speak of positive value, but if the reaction is negative, we speak of negative value. In Pavlov's experiments, dogs "drew themselves" to food, but much more to meat than bread (1952, p. 36). Therefore, we can state that they found meat more valuable than bread. On the contrary, when the sight of a strange man evokes an aggressive reaction in a dog and makes it snarl and attack, we conclude that the stranger must have a negative value for the animal. 
Both forms of value do not have to come in pairs: for example, a hungry sparrow may voraciously devour caterpillars which will make it die, while wild animals will react negatively to a human for a long time during the taming process even though the human does not pose any threat to them and in fact provides food and water and cleans their cage, etc. Still, in many cases we can conclude that animals react as if they "knew" what is biologically beneficial or harmful for them; thus, the question arises as to what orientation in value really is.

The reflection mechanisms discussed above do not suffice to answer this question because orientation in value cannot be considered a form of "reflecting value". First and foremost, as is commonly known, no organism possesses separate "analyzers of value"; secondly, even objective biological values cannot be treated as ab solute features of objects, such as color, taste or scent. The value an object has for an individual is a relative feature that depends on the relation between the properties of the object and the properties of a given organism. Depending on what the organism is like, one and the same object can have a positive or negative value for it or can have no value at all, thus the organism is indifferent. For example, meat has value for a cat, but is insignificant for an herbivorous goat; long immersion in water is beneficial for a fish, but poses a deadly risk for a land animal, etc. Simultaneously, the value a certain object has for an individual is closely related to the features of the object which are "necessary" or, on the contrary, harmful to the animal. These features are the only possible indications of value, which leads to the conclusion that orientation in value, which is not a reflection process itself, must happen, so as to say, "inside" the reflection of objects and phenomena which are "appropriate" or "inappropriate" for the individual. In other words, while reflecting indications of value, an organism must already include value itself in the reflection process. How can we imagine this fact as a nervous process and define it in terms superior to those used in the psychology of consciousness?

Unfortunately, physiology, to which we owe the core information about the reflection process, has little to say in this matter. Some information can be attributed to Pavlov, who, when investigating adaptation to the environment, had the most opportunities to come across this question. However, this information is limited to unrelated casual observations and from which we must reconstruct our own neurological model of what we would call "orientation in value". Primarily, the starting point should be the conception of orientation in the environment that Pavlov, as I have mentioned before, tended to use. The most elaborate statement in this respect 
distinguishes two levels of orientation in the environment in animals, presenting functions of two separate, central "action systems"7: the subcortical reflexive-unconditioned system and the cortical reflexive-conditioned system. These are two distinct yet functionally closely connected systems of adaptation to the environment, that is "two instances of complex relations between an organism and the environment": the former governs instinctive, inherent adaptive activities, while the other manages activities developed over the course of an individual animal's life. From Pavlov's remarks it follows that both systems enable an individual's orientation in the environment, with the reservation that the inherent orientation is "limited" due to the small number of stimuli by which the animal can orient itself naturally. Pavlov describes acquired reflexive-conditioned orientation as "extensive" because it concerns a considerable number of conditioned stimuli. In other words, experience significantly increases the number of stimuli by which the animal can orient itself and to which, as a consequence, it can adapt its reactions (1952, p. 497). Pavlov made but a general statement about this; therefore, we should ask what, according to him, this inherent and acquired orientation in the environment can mean. Let us try to solve this problem by considering a concrete example from his experiments. Described during a lecture delivered in Madrid, one of the first experiments involved pouring a black-stained acid into a dog's mouth as a result of which, after some time, the very sight of black liquid made the animal salivate because the color black was now associated with a sour taste and had become a conditioned stimulus. What makes this experiment significant is that the unconditioned stimulus, i.e. the sour taste, and the conditioned one, i.e. the black color, are properties of one and the same substance, i.e. the acid. After conditioning, both elicit salivation, but each of them works according to a different principle: the taste arouses the salivary glands naturally, whereas the black color triggers a reaction because it regularly accompanies the sour taste. This type of experiment led Pavlov to formulate a law governing the learning of conditioned stimuli: "Insignificant features of an object become stimuli for organs (in this case, salivary glands) only when their effect on the stimulated surface of the organism coincides with the effect of significant properties" (1952, p. 45). The notion of "significant" and "insignificant" properties was explained in Chapter I of the Lectures: according to Pavlov, "significant" properties are those that by themselves are beneficial for or harmful to the animal. These are primarily "contact" features due to which the object enters into a direct reaction with the organism, e. g. the taste of food, the sharpness of teeth and claws tearing an animal's skin, etc. On the contrary, "insignificant” properties are neither

\footnotetext{
${ }^{7}$ For the concept of action system see Ivanov-Smolensky, 1951, pp.33-35.
} 
beneficial nor harmful by themselves but announce or precede significant ones, due to which they elicit a similar anticipatory, replacement reaction. For Pavlov, these were mostly "distance" stimuli: sights, scents and sounds (1951, p. 27). By combining this information, we must conclude that, for Pavlov, "orientation in the environment" means basically orientation in value; this sometimes happens on the basis of "significant" properties of an object that play the role of inherent indications, such as a sour taste, or sometimes on the basis of "insignificant" properties that play the role of learnt indications, such as the color black. Undoubtedly, inherent orientation in value is "limited": an animal, for example a dog, would have a slight chance of survival if it oriented itself in the nutritional value of an object only on the basis of its taste, or if it defended against an enemy only when it felt the enemy's claws tearing its skin. Certainly, the ability to orient oneself in the value of an object on the basis of its indications of distance extends the scope of orientation and elevates the entire adaptation activity of an animal to a higher level.

Thus, it follows that orientation in value is a significant component of conditioned and unconditioned reflexes, which means that the physiological model of this phenomenon should be found in the mechanism of reflex processes. This search can be made simpler by focusing primarily on the mechanism of unconditioned reflexes. Even though the thesis is not obvious, Pavlov's experiments demonstrate that inherent orientation in value plays a major role in the adaptation of animals to the environment, while acquired reflexive-conditioned orientation does not expand the scope of value but only enriches the animal's experience with new orientation indications whose effect boils down to anticipatory activation of inherent orientation in the value of a given situation. Therefore, if we wish to reconstruct the Pavlovian model of orientation in value, we should rely on an analysis of reflexive-unconditioned processes in which the model must exist in its basic, purest form.

\section{3. "Basic tendencies" of an organism and inherent orientation in value}

As we said earlier (see p. 97), Pavlov distinguished a guiding process in the reflexive-unconditioned mechanism which made an animal sensitive to certain stimuli and forced it to react in a particular way; he called this process the "basic tendency of an organism". Let us investigate this term more closely. 
The term "tendency" is derived from psychology. It is equivalent to "aspiration", a term used in the psychology of consciousness. For scholars such as Tieplov, drives and desires (Tieplov, 1950, p. 162) are, in general terms, "motivational" experiences that push one to perform certain actions towards certain objects. However, Pavlov uses this term to denote not experiences but a specific type of nervous process. The following conception of "tendency" can be presented on the basis of various statements he made in different papers.

First and foremost, tendencies are a function of the reflexive-unconditioned centers. As I have already mentioned, Pavlov often situated these centers in subcortical nuclei, located in the direct vicinity of the cortex; however, after considering all his remarks on this topic, we must conclude that this statement is imprecise. The whole theory indicates that the centers are two-level structures found not only in the subcortex but also represented in the cortex. In the paper on the "digestive center" (1910) Pavlov already clearly formulated the opinion that this center comprises a cortical element besides the subcortical part, "in the form of taste centers" (1952, pp. 141-2). In other words, in line with this view, contact-based, unconditioned nutritional stimuli are received by the cortex and elicit an inherent reaction through cortical taste cells. This thought was generalized in the Lectures to refer to all unconditioned stimuli in the statement that "if the brain is intact, all stimuli evoking unconditioned reflexes in animals primarily reach specific cells of cerebral hemispheres" (1951, p. 45), while in the Croonian lecture delivered a year after the publication of Lectures, these cortical components of reflexive-unconditioned centers were described as "active points representing unconditioned reflexes" in cerebral hemispheres (1952, p. 395). By this token, reflexive-unconditioned centers comprise not only subcortical nuclei but also certain parts of the cortex; however, it should be presumed that the subcortical and cortical components must be connected to inherent nerve routes within each center. Otherwise, it would be impossible to understand how unconditioned stimuli received by the cortex could elicit certain reactions from birth that should basically be comprehended as the function of subcortical cells that governs inherent motoric function and secretion. ${ }^{8}$

In the bipartite, subcortical-cortical structure of the reflexive-unconditioned center described above, both components are engaged in the reception of stimuli, but the subcortical component plays a different role than the cortical one. As follows from Pavlov's remarks, the cortical cells that are a

\footnotetext{
${ }^{8}$ The fact that conditioned reflexes are present in animals whose cerebral cortex has been removed allows us to assume that the receptive role of the cortical element is performed by phylogenetically older subcortical cells (Head), which may be inactive in a normal animal in which reception is governed by the cortex, which is a hierarchically superior layer.
} 
part of the center perform the core function of receiving unconditioned stimuli; therefore, they are sensory, receptive cells. Pavlov understood the subcortical parts of the centers as certain motors that energize the cortex. He formulated this thought in various ways, e.g. by claiming that "subcortical centers determine the state of activity of cerebral hemispheres to a larger or lesser degree" (1952, p. 420) or that "the parts that are closest to the subcortical regions send from their centers a mighty stream of energy to the cortex, thus maintaining its tension" (ibid. p. 462), etc. Pavlov studied the function of subcortical centers more thoroughly in his work on hysteria (1952, p. 490). He made it explicit that the primary activity of subcortical nuclei is the production of "basic tendencies of the organism: digestive, sexual, aggressive, research-related, etc." However, it cannot be said that the "tendencies" are exclusively the function of the subcortex because, according to Pavlov, they originate in the cerebral cortex or-as Pavlov used to say-in "cerebral hemispheres". Pavlov writes that "tendencies originate under the influence of external or internal excitation. The activity of a point or circuitry in the cerebral hemispheres corresponds to a tendency. Under the influence of emotions and as a result of irradiation from subcortical regions, such a point receives a strong 'charge”. Further, Pavlov studies how the "charge" (probably the stimulating one), which is concentrated in a "certain point or circuitry" of the cortex, inductively inhibits the rest of the cortex in hysterics, which explains the "emotional" behavior of the patients. If we were to extract objective physiological content from this free semi-psychological and semi-physiological term, the process corresponding to "tendency" could be described with three conceptions:

1. It is an "activity of a certain point or circuitry of cerebral hemispheres";

2. It originates "as a result of irradiation from subcortical regions", which thus "charge" a given cortical region, that is probably induce in it a state of excitation;

3. The whole process of "charging" the cortex from subcortical centers, which corresponds with "tendency", can be elicited by either an external stimulus or "under the influence of internal excitation".

Therefore, tendency is a joint activity of both components of the reflexiveunconditioned center. It is produced by the subcortical part of the center, but the place where tendency originates is the cortical, sensory part that constitutes the organ receiving unconditioned stimuli. In other words, when a particular tendency appears under the influence of either external or internal factors, both the subcortical and cortical and the receptive and performing parts of the whole center become excited. This excitation causes a state of excitation in the center and prepares it to reflect certain stimuli 
and react to them with a specific action or, to put it in other words, it dynamically directs the action of the central nervous system and the ensuing activities. This must be the reason that Pavlov described it with the term "tendency" derived from psychology.

Applying the notion of tendency to the problem of perceiving value, we can formulate an opinion on how one can imagine the process of orienting oneself in the value of objects on the basis of these conceptions. Namely, this process can be understood as a kind of "collision" of two waves of stimulation in the sensory part of a certain reflexive-unconditioned center: the first wave flows from a particular subcortical nucleus inside and is identical to a relevant tendency, the second is elicited from the outside by a particular unconditioned stimulus. Thus, there are two types of this process: depending on the manner in which tendency appears, it originates either from the inside or under the influence of external excitation.

An example of the first type of perception of value may be orientation in the nutritional value of an object, which is closely related to the tendency that appears depending on internal factors. In this case, we must assume that as a result of metabolic processes, particularly the change in the chemical composition of blood (Pavlov speaks of "hungry blood", see e. g. 1952, p. 134), a subcortical component of the digestive center becomes stimulated and starts to irradiate on its cortical representation, i.e. on the taste region of the cortex, following which the stimulation focus originates here, meaning the cortex is oriented at, or sensitized to, taste and alimentary stimuli. If an appropriate external stimulus affects the sensitized cortex, the two waves of stimulation merge in the cortical part of the center: one of subcortical origin and the other from the exteroreceptor. In this situation, the stimulus is consistent with the tendency, i. e. it becomes valuable from the perspective of the present condition of the organism. When the animal is fully satiated, the digestive tendency does not appear, and taste stimuli do not elicit any reaction because the animal will not notice their value due to the lack subcortical excitation. ${ }^{9}$

A similar merger or collision of excitations should happen in the other type of process that occurs when the reflexive-unconditioned center becomes irritated from the inside, as in the case of a dog that, when suddenly touched, updates the aggressive reflex, snarls and tries to bite. The process of the origin of the aggressive tendency can be imagined in a two-fold manner: it

\footnotetext{
${ }^{9}$ For the sake of simplification, interoceptive excitations related to the irritation of stomach walls were disregarded in this perception. Undoubtedly, interoception is a vital factor but is not identical to the very alimentary "tendency" elicited under the influence of irradiation from the subcortex on the cortex.
} 
is possible that on its way to the cortex the nerve impulse that is elicited by a stimulus first excites the subcortical component of the aggression center and, owing to irradiation, immediately produces a relevant tendency in the cortex so that upon reaching the cortex the exteroceptive excitation comes across the excitation charge of subcortical origin. But it can also be assumed that the tactile impulse goes only through the subcortical charging stations to the cortex, where it first elicits excitation that next goes along inherent nervous routes down towards the subcortical part of the center, which then begins to irradiate on the cortex as a result of which the excitation from the subcortex joins with the external excitation. In both cases, the two kinds of excitation collide in the cortex, which-in line with the outlined conceptdetermines the orientation in the value of the stimulus, in this case orientation in the danger that a sudden touch poses for a dog.

Only such perception of orientation in the value of objects helps us comprehend how, not being a separate form of reflection, this process can be associated with the reflection of certain features of an object, thus creating orientation in value. When a tendency collides in the subcortical cortex with the reflection of a given factor functioning as an unconditioned stimulus, for example the taste of food, the very factor is already positively or negatively reflected in line with the tendency depending on the nature of the active tendency. Thus, the nervous system does not perceive the value of an object separately: it perceives it in the reflection of a given stimulus presenting an indication of value, in a positive reflection if the stimulus is consistent with an offensive tendency, and in a negative reflection if it is consistent with a defensive tendency.

Now let us return to the previous remarks on how we can understand the "value" a given situation may have for an animal. It is obvious that by observing a behavior we only determine the behavioral value that presently can be specified as the consistency of the features of an object with the tendency that is active at a given moment in the animal. We can compare this behavioral value with the objective biological value of the situation and conclude whether the animal oriented itself in the situation correctly or made a mistake. The fact that orientation in the value of objects found in and the phenomena occurring in their natural environment is usually typical in animals implies that the reflexive-unconditioned mechanisms correctly perform the role of guardians watching over the biological interests of an individual. But there will also be cases in which we will be limited solely to the behavioral value. This will happen everywhere the animal follows not "self-preservation" but "species" tendencies (see p. 98). Obviously, we could try to search for an "objective value" even here in the benefits a given behavior brings the species; but then, by ascribing to an animal the ability of 
orienting in species benefits, we are heading towards some biological metaphysics. In this case, it is better to start with the fact that an animal displays tendencies typical of the species, the realization of which contributes to maintaining balance in the nervous and endocrinologic systems-just as do the self-preservation tendencies protecting its whole organism. "Species values", for example the well-being of offspring, would thus be "personal" values for animals even though we cannot provide an objective biological benefit they have for an individual organism when it comes to preserving its health and sustaining its life.

\section{The conception of tendency as a term used in psychology of adaptation}

It follows from the above considerations that in order to specify what orientation in the value of an object is, we should use another term besides "reflection", namely, the conception of tendency or the equivalent conception of need (see p. 98). This is the second major term in the psychology of adaptation. Tendencies themselves are not a part of the cognitive process which is the topic of these remarks, but they have to be taken into account in the terminological analysis of "cognition" because they provide reflection with a positive or negative character that is as essential for the process as the cognition of the value of objects. Thus, let us devote some more attention to this term, firstly, in order to emphasize its significance and, secondly, in order to separate it from some other notions that are synonymous but not identical to it.

Primarily, it must be underlined that, despite being defined by Pavlov in physiological rather than psychological categories, "tendency" is a psychological term, not a physiological one. The word means "a certain direction" (in Latin tendo denotes tautening the bow while aiming it towards a particular direction and metaphorically refers to pursuing a goal) and the nervous process described with this word by Pavlov pertains indeed to an organism directing itself at particular components of the environment. If we define this term in this way, we will describe it in psychological terms by showing the relation between the process discussed above and the objects of the external world. Defined so, "tendency" means being oriented towards a positive or negative reaction to certain external stimuli-it becomes a certain positive or negative "attitude" of the nervous system and, consequently, the "attitude" of the whole organism towards particular phenomena. Therefore, tendency constitutes a term superior to "aspirations", "desires", "needs" and other similar terms used in the psychology of consciousness. Understood in the psychological manner described above, this term plays a vital role in research into adaptation because it allows us to 
explain and understand the direction of behavior "towards" and "from" an object. If we assume that this direction is usually consistent with the objective biological value the objects from its environment have for the animal, we must acknowledge that tendencies comprise a vital component of the mechanism of adaptation to the environment and deserve to be considered. This term is not an unnecessary "doubling" of physiological notions. The physical mechanism of the irradiation of excitation from the subcortex to the cortex does not yet allow us to understand the role this process plays in the mechanism of adaptation to the environment, just as the "play" of excitation and inhibition in the cortex does not suffice to understand the adaptive meaning of the reception processes. Only the terminology describing these processes in relation to the environment, i.e. describing them psychologically, makes it possible to explain their exact role in the relations between an individual and its environment. As material nervous processes, tendencies undoubtedly possess their own physiological mechanism and the task of science is to study this aspect of theirs; still, their psychological description cannot be replaced with a physiological one, just as a physiological description of, for example, digestion cannot be replaced with a chemical definition.

However, while introducing the term "tendency" to behavioral psychology, we need to be extremely careful not to confuse it with other similar conceptions. Considering the considerable ambiguity of psychological terms, such confusion seems unavoidable. Proof of this is the polemic between Rubinstein and American psychologists on the use of the term "tendency" to explain animal behavior (see Rubinstein, 1954, p. 181). Rubinstein, Hilgard, Marquis and others are of the opinion that in order to explain the "direction" of behavior, it should be assumed that "there exist internal impulses (in the form of drives, instincts and tendencies) concealed behind reflexes that are treated as "mechanisms" and which allegedly steer the activity of these reflexive mechanisms in the direction the organism needs", thus "accusing Pavlov of mechanicism on the basis of the fact that he does not assume motivation is hidden behind the mechanism of reflexes". Trying to defend Pavlov against this accusation, Rubinstein states that in the Pavlovian understanding, reflex is not a mechanical "blind" reaction but an activity of life aiming, according to Pavlov, to search for "living conditions that are essential and indispensable for an animal and that constitute unconditioned stimuli" in the changing environment (quote from Dwadzieścia lat badań, 1952, p. 408). With such an understanding of reflex, "a stimulus elicits appropriate reflexive reaction in the organism because... it replies to the requirements imposed by the organism on the environment, to the needs of this organism", and not because a separate motive pushing it towards this stimulus affects the organism. Thus, Rubinstein concludes that "developed 
by Pavlov, the study of reflexive action does not require and cannot be reconciled with any motive, drive or impulse hidden behind the reflex deep in the organism that would activate this reflex mechanism in a mysterious way" and the psychologists who attack Pavlov for failing to consider motivational processes "do not understand the genuine Pavlovian research and distort it”.

It seems we are facing a double terminological misunderstanding here: on the one hand, on the part of Pavlov's critics who-as Rubinstein rightly underlines-wrongly understand the Pavlovian conception of reflex and think that a "reflex" excludes any behavior-motivating tendency, and, on the other hand, on the part of Rubinstein himself who, adopting the American understanding of "motive" as a process joining a reflexive action "from the outside", reaches the false conclusion that the conception of tendency is generally inconsistent with the reflexive theory of adaptation. When it comes to the first issue, the accusation that behavioral motivation is not taken into account is probably based on the wrong association between the Pavlovian conception of reflex and the traditional understanding of "reflex" as a stereotypical, implastic activity steered by inferior nervous centers. It seems obvious that if we wanted to limit all the adaptation activity of animals to reflexes understood in this way, we would have to assume the existence of a superior motivational mechanism that steers this "blind" reflexive activity towards the direction the organism needs. However, the Pavlovian understanding of reflex is much broader than the traditional definition of the term because, besides such "inferior" reflexes, it includes a complex, instinctive activity and learnt reactions in which the cortex plays the major role (1951, pp. 22-27). Pavlov did not study inferior reflexes, such as spinal ones, extensively, limiting himself to mentioning that physiologists were better and better at understanding the mechanism of "this as if automatic activity of an organism" (ibid., p. 22) and focused all his interests on "superior" reflexes, i.e. activities that were not commonly and traditionally considered reflexes. Pavlov does not state that these reflexes are deprived of plasticity or motivation, proof of which is the fact that he distinguished the "core tendencies of the organism" as a separate component of the reflexive mechanism of instincts. Depending on their function, these core tendencies can be described as the "motives" of the reflexive-unconditioned action. As Rubinstein rightly remarks, these are not motives joining a "blind" reflexive action "from the outside" but reflexive tendencies-components of the mechanism of complex involuntary movements, i.e. instincts. The difference between the Pavlovian and non-Pavlovian conceptions of behaviormotivating tendency would be that Pavlov understands tendencies as an integral component of broadly-understood reflexes. Other authors consider them as a separate internal process that conditions, besides other factors, 
behavior, which-in line with the traditional understanding of the term "reflex"-does not have reflexive features ascribed, so eventually the whole difference is purely terminological.

However, taking this into consideration, we should also conclude that the reflexive theory of adaptation does not exclude behavior-motivating tendencies; on the contrary, it requires that they should be accepted as a vital element of the reflexive mechanism. Rubinstein's statement that "according to Pavlov, the very connection between the stimulus and a reflex reaction of the organism depends on the biological significance of stimuli, that is on their relation with the needs of the organism" is true but does not reflect the entirety of Pavlov's views. Let us pay attention to the fact that the conception of need formulated by Rubinstein most probably does not mean any process that would determine a certain behavior of an animal, but some objective properties of the organism related to its biochemical and biophysical structure, due to which it needs certain external conditions in order to exist. In this sense, it can be said that, for example, fish "need" an aquatic habitat to exist or carnivorous animals "need" meat as food, etc., i.e. neither could live if they did not find these "needed" objects in their environment. Rubinstein emphasizes that reflexes are the tool used by an animal to obtain these "needed" objects, as a consequence of which the very conception of reflex is sufficient to comprehend the adaptive activity of the organism. However, if we understand "need" as a specific structure of an organism, we must enquire about the processes that make it possible for this structure to provide itself with everything it needs to exist through self-regulation. It is not enough to say that this happens simply due to reflexes as this only leads to another question: What is the mechanism of this reflexive adaptation activity which results in each violation of internal balance of an organism eliciting an act aimed exactly at what is needed to remove the disturbance? In order to answer this question Pavlov introduced the conception of tendency as a factor operating within the framework of the reflexive mechanism and directing the organism to react to the environment in a particular way.

Another misunderstanding can be confusing the conception of tendency with some meanings of the term "need". As we have said, for Rubinstein this conception signifies a certain structure, or a permanent property of an organism, but it is commonly understood as the process stimulating the organism from the inside to undertake some specific activity, i.e. the phenomenon of the "tendency" type. The conception of need is frequently used in psychology. To provide an example, I will list the works of Katz, Lewin, Szymański and many American publications in which this term (need) plays—as I have already mentioned (p. 98)—a major role. Still, not 
all meanings of this term can be simply associated with "tendency". For example, Lewin understands "needs" as "spiritual forces" (seelishe Kräfte) which are a "result" of processes such as "drives" or "internal needs" (zentrale Wollungen), but which are not identical to them (Lewin, 1926, p.59). Seemingly, Lewin is a proponent of functional dualism and considers need as a certain state of consciousness that is "derived" from organic processes, while in Pavlovian meaning, "tendency" is a material nervous process. Undoubtedly, in certain cases this understanding of tendency acquires a conscious superstructure and transforms into the desire to react to a particular stimulus with a given activity, but then it does not become some vague "spiritual force".

At this point, we should devote more attention to another way of understanding "need" which is based on Pavlovian ideas, i.e. the definition propounded by Selivanov, according to which "all study of conditioned reflexes" is nothing but a study of "causes and motives of behavior" (Selivanov, 1954, p. 344). However, in order for it to be exploited by a psychologist, it is necessary to use the notion of need (loc. cit., pp. 345-6). Nevertheless, Selivanov rejects the ways in which this term is understood in psychology, particularly-and he must mean Freudianism here-the "anti-scholarly, autogenetic conception in line with which need is an inner force that is independent of external conditions and matter in general, and which derives fatalistically from the depth of the body, sexual cells and genes and determines all vital activity of the organism". Instead, he proposes a different understanding of the term based on the biological views of Michurin. Selivanov writes: “according to Michurin's biology, needs as stimuli to a vital activity are typical of all living creatures, not only animals, but also plants. The internal essence of the vital activity of each organism is metabolism. Every act of life that leads to dissimilation, that is the burning of the cells of a living organism, generates energy and consequently the need for assimilation, i.e. the need to reproduce new, living cells from the external environment. Thus, the conception of need reflects the constant disturbance of this balance in a metabolism, which causes the process of dissimilation that counterbalances the process of assimilation" (loc. cit., p. 346). Needs are not fixed factors, but they depend on external conditions which-by changing and "influencing organisms-reorganize these functions of the organism and rebuild the type of metabolism, thereby generating new needs of the organism corresponding to the changed conditions of the external environment" (loc. cit., pp. 346-7). The tool regulating the behavior of an organism to satisfy these "needs of assimilation" is the brain and Pavlov's studies discovered the laws according to which this "adaptive activity of the brain" happens (loc. cit., p. 347). Initially only a process of chemical changes taking place in the organism, need becomes the "stimulus 
of action" owing to brain activity: elicited with dissimilation, the changes in the cells of the organism are signaled introceptively to the brain "and this is one aspect of need as a stimulus of action". Another extremely important aspect of need which has the form of impulses received by the brain from the external environment is based on external conditions in objects which can satisfy the needs of an animal in terms of food, self-preservation, reproduction, etc. In highly developed animals, the external meets the internal in the cortex. In the process of a complex, analytical and synthetic activity, in the relation between the external and internal signaling systems there develops a stimulus which, expressing itself physiologically in the form of a dominant feature, is a direct cause of the act of behavior" (loc. cit., p. 348).

It would probably be difficult to precisely define the conception of need with which Selivanov covers the processes happening in both animals and plants. Limiting this task only to animal needs, we should state that need is a process consisting of changes that happen in the substance of an organism which disturb its balance under the influence of dissimilation. This process also comprises a nervous "stimulus" elicited in the brain by these changes that pushes the animal to pursue an action to remedy deficiencies. Comparing this meaning of "need" with Pavlovian "tendency", we should first and foremost emphasize that tendency is a purely nervous process that originates in the cortex as a result of irradiation from the subcortex, whereas Pavlov would treat the use of cells under the influence of dissimilation as an internal factor which updates the tendency, but which is not a component of tendency itself. Thus, tendency should be associated with Selivanov's "stimulus" rather than the whole "need". However, disregarding this difference, we should notice that "tendency" is much broader than "need", even when understood as a stimulus to act, primarily because Pavlov assumes the existence of defensive tendencies which are elicited not from the inside but from the outside and which cannot be explained with dissimilation and considered to be "assimilation needs". In the Pavlovian understanding, "tendencies" are nervous processes, components of reflexive mechanisms and guardians of the balance of the organism against all disturbances from both the inside and outside, owing to which they can provide the animal with comprehensive adaptation to the environment.

Undoubtedly, the above remarks do not cover all possible misunderstandings related to the conception of tendency. I have limited myself to discussing primarily the definitions proposed by Rubinstein and Selivanov because these authors try to exploit Pavlovian conceptions for the sake of psychology, owing to which it is particularly probable that they would confuse the conceptions they use with the term "tendency". 


\section{Orientation in action}

Let us proceed to the other component of orientation in the environment and consider how this process could be discussed not from the point of consciousness but from its nervous background. Pavlov's research provides us with a convenient physiological model also in this respect.

Although basic Pavlovian experiments relied on salivary reflexes in which orientation in action was excluded intentionally, Pavlov had been interested in the motoric element of adaptation almost since the very beginning of his work on superior nervous activity. He had already tried to formulate a physiological theory of acquired motoric reactions in 1907 (1952, pp. 1034), and the experiment conducted by Krasnogorsky in 1911 to demonstrate that part of the motoric region in the cerebral cortex of a dog is a cerebral pole of the motoric analyzer was the first attempt at experimental examination of these reactions. Pavlov studied this issue in more detail as late as in 1930, seemingly inspired by the experiments performed by Konorski and Miller first in Warsaw and then continued in one of Pavlov's laboratories. Konorski and Miller altered and developed the technique used by Krasnogorsky, which led them to conclude that if a passive movement of a dog's leg were continuously combined with a neutral external stimulus, and if this combination were regularly reinforced with food, it would elicit in the animal not only a salivary conditioned reflex in reaction to the passive movement of the leg as a proprioreceptive alimentary signal-as in Krasnogorsky's experiment-but also, after some time, it would cause the dog to actively lift its leg in reaction to a given external stimulus. It could be stated that in these experiments researchers showed the animal a movement that constituted a peculiar way of winning food in experimental conditions. The fact that the animal appropriated the movement and learnt to perform it actively demonstrates that it oriented itself in its significance as a manner of effecting the alimentary tendency adapted to the situation. Moreover, the experiments reveal that the external stimulus, which was constantly associated with the leg movement, became an indication pointing to action: it "showed" the animal that in order to obtain food, it had to lift its leg. In other words, it is a simple, laboratory example of "active" orientation in the environment: it is not only orientation in the value of the alimentary unconditioned stimulus intended for the animal, but also orientation pointing to action that constitutes the manner of obtaining this value in a given situation.

On the basis of the experiments performed by Konorski and Miller and the research conducted by Skipin, Petrova, Podkopaev and others (see Mayorov, p. 236f.), Pavlov proposed a certain conception of the mechanism of acquired movements, described extensively in the paper under the Polish 
title Fizjologiczny mechanizm tzw. ruchów dowolnych (1952, pp. 579-82). The disquisitions presented in the paper are complemented with Pavlovian Wednesday lectures on this topic, particularly the two delivered on 10 October and 14 November 1934 (Pawł. Sr., vol. II, 480-90, 536-39). At this point, I will not analyze or interpret Pavlov's statements in detail but limit myself to describing his hypotheses to the extent needed for the issues discussed herein.

The core of the conception is the thesis that the movements performed by an animal are proprioreceptive stimuli received with a separate movement analyzer whose peripheral endings are muscle and joint receptors and whose cerebral pole is located in the superior layers of the motoric region, the latter being in line with Krasnogorsky's experiment (see Pawł. Sr., vol. II, p. 481). It follows from this that each movement of the legs and the torso is registered in the cerebral cortex. Due to the commonly known fact that the cortex is also governed by the principle of spatial, and certainly temporal, relevance of stimuli as well as receptor and central processes, every time the cortical processes are elicited by certain movements of the body, they create configurations constituting proprioreceptive reflections of these movements. By this token, it can be stated that animals know (meaning: reflect) not only the environment, but also their own movements.

The second Pavlovian thesis claims that while one learns new movements, a given activity performed by one in a particular situation by accident or as a passive movement and then "reinforced" with an unconditioned stimulus-for example an alimentary one-is associated with both this stimulus and the situation, as a result of which the very reflection of the external situation later updates the proprioreceptive "reminder" of this action in the animal. Moreover, Pavlov assumes that the proprioreceptive cells which comprise the cerebral pole of the movement analyzer possess not only afferent connections with certain muscles but are also connected with them efferently through the nerves joining these cells to the executive cells found in the inferior layers of the motoric region (1952, p. 579). Consequently, the cortical "reminder" of the action elicited through a situation must trigger certain muscles along these efferent routes, i.e. it must elicit the performance of a relevant movement. ${ }^{10}$

\footnotetext{
${ }^{10}$ I do not discuss here the technical problems that the researchers came across while conducting experiments in triggering motoric reactions and the difficult issue of the mechanism of learnt defensive movements that Pavlov merely touched upon but never solved. (See Mayorov, 1948, pp. 347-8).
} 
Thus, orientation in action can be comprehended as an association between the exteroreceptive reflection of a situation and the proprioreceptive reflection of an action that is strictly connected to the orientation in the value of an object, for example the value of an unconditioned stimulus intended for an animal. The external situation, or its component that constantly accompanies the performance of a movement and that is reinforced with it, constitutes the indication pointing to the action that must be performed in order to obtain this value.

The scheme of orientation in the environment that results from the experiment of Konorski and Miller is extremely simplified, appropriately to the very simple experiment on which it is based. Undoubtedly, orientation in action is normally a much more complex process. For example, let us consider Köhler's experiment in which a chimpanzee learnt how to retrieve with a stick a fruit hanging outside the cage. In this case, the sight of the fruit is an indication of the value of the bait, while the sight of the stick plus the distance separating the ape from the fruit is a complex indication pointing to the action it has to perform to retrieve the fruit. However, here the process of orientation in action is different than in the experiment conducted by Konorski and Miller. The action required to perform the tendency here is not limited to a single movement of a limb but consists of a series of manipulative movements (excluding locomotive movements), starting with grasping the stick and ending with retrieving the fruit. By this token, orientation in this action does not consist of remembering a single movement once under the influence of a given indication pointing to action, but has to happen while the whole action is performed: the sight of the stick reminds the ape of the movement of grasping, as a result of which the animal grasps the stick, but this changes its situation and triggers new indicators orienting the animal to the fact that it should reach beyond the cage towards the fruit. The sight of the stick approaching the fruit orients the animal to further movements, etc. until the ape can catch the fruit with its hand. Contrary to the artificially simplified experiment conducted by Konorski and Miller, the process of orientation in action on the basis of constantly changing visual and most probably tactile indications with which the stick impacts the ape's hand continuously accompanies the movements and directs them. However, this complex orientation in action should consist of associating the changing indications with relevant movements so that they create a certain chain of associations, such as the simple connection that constitutes the basis of a single limb movement. The analysis of particular forms of orientation in action is a task for concrete studies in this respect. Here, the point is the very principle governing this process which, in the light of the conception discussed above, boils down to the association between the external indication of orientation and the reflection of action. 
The above considerations seem to indicate that not only "reflection" but also the entire "orientation in the environment" can be regarded as terms superior to the relevant terminology used in psychology of consciousness. Up to a point this is confirmed by the fact that we can provide relevant physiological "models" for both orientation in value and orientation in action. Based on Pavlovian ideas, which are particularly convenient for such analyses, these models cannot claim the right to be called proven physiological truths, as is true in the case of most statements concerning the cerebral reflection of external stimuli. They are simply some tentative hypotheses and their value for our considerations lies in demonstrating that it is not unreasonable to see names of nervous processes in terms such as "orientation in value" and "orientation in action" and use them irrespective of whether these processes are conscious or not. However, the veracity of these physiological hypotheses is not a sine qua non for using these terms. Even if further progress in physiological research demonstrated that the physiological mechanism of instinctive tendencies is slightly different than Pavlov imagined, or that the proprioreceptive representation of acquired movements should be sought not only in the motoric region of the cortex, the very conception of orientation in the environment would not be diminished and research results in this field would remain valid, obviously assuming that they were sufficiently grounded in facts.

\section{References}

Dembowski, J. (1946a) Psychologia zwierząt. Warszawa: Czytelnik.

Iwanow-Smoleński, A. G. (1951) Zarys patofizjologii wyższych czynności nerwowych. Warszawa: Państwowy Zakład Wydawnictw Lekarskich.

Ivanov-Smolensky A. G. (1954) Essays on the patho-physiology of the higher nervous activity: According to IP Pavlov and his school. Moscow: Foreign Languages Publishing House.

Lewin, K. (1926) Vorsatz, Wille und Bedürfnis. Berlin: Springer.

Lewin, K. (1951). Intention, will and need. In D. Rapaport, Organization and pathology of thought: Selected sources (pp. 95-153). New York: Columbia University Press.

Mayorov, F. P. (1948) Istorija uczenija ob uslownych riefleksach. Moskow.

Pavlov, I. P. (1951). Wykłady o czynności mózgu. Translation: S. Miller. Warszawa: Towarzystwo Wydawnicze "Rój".

Pavlov, I. P. (1952). Dwadzieścia lat badań wyższej czynności nerwowej (zachowania się) zwierząt. Translation: T. Klimowicz. Warszawa: Państwowy Zakład Wydawnictw Lekarskich. 
Pavlov I. P. (1973) Twenty-year Experience of objective study of higher nervous activity (behavior) of animals. Lectures on Conditioned Reflexes. London: Pinter Pub Ltd.

Rubinstein S. Ł. Nauka Pawiowa a zagadnienia psychologii. In Nauka Pawłowa a filozoficzne zagadnienia psychologii: zbiór artykułów. Translation: Z. Babska (pp. 171-201). Warszawa: Państwowe Wydawnictwo Naukowe.

Rubinstein, S. L. (1952) The Pavlov doctrine and Problems of Psychology. In the Collection: The Pavlov Doctrine and Philosophical Problems of Psychology. Moscow.

Selivanov W. I. (1954). Przyczynek do zagadnienia tak zwanego zachowania się dowolnego. In Nauka Pawłowa a filozoficzne zagadnienia psychologii: zbiór artykułów. Translation: Z. Babska (pp. 325-357). Warszawa: Państwowe Wydawnictwo Naukowe.

Teplov B. M. (1950) Psychologia. Warszawa: Nasza Księgarnia. 